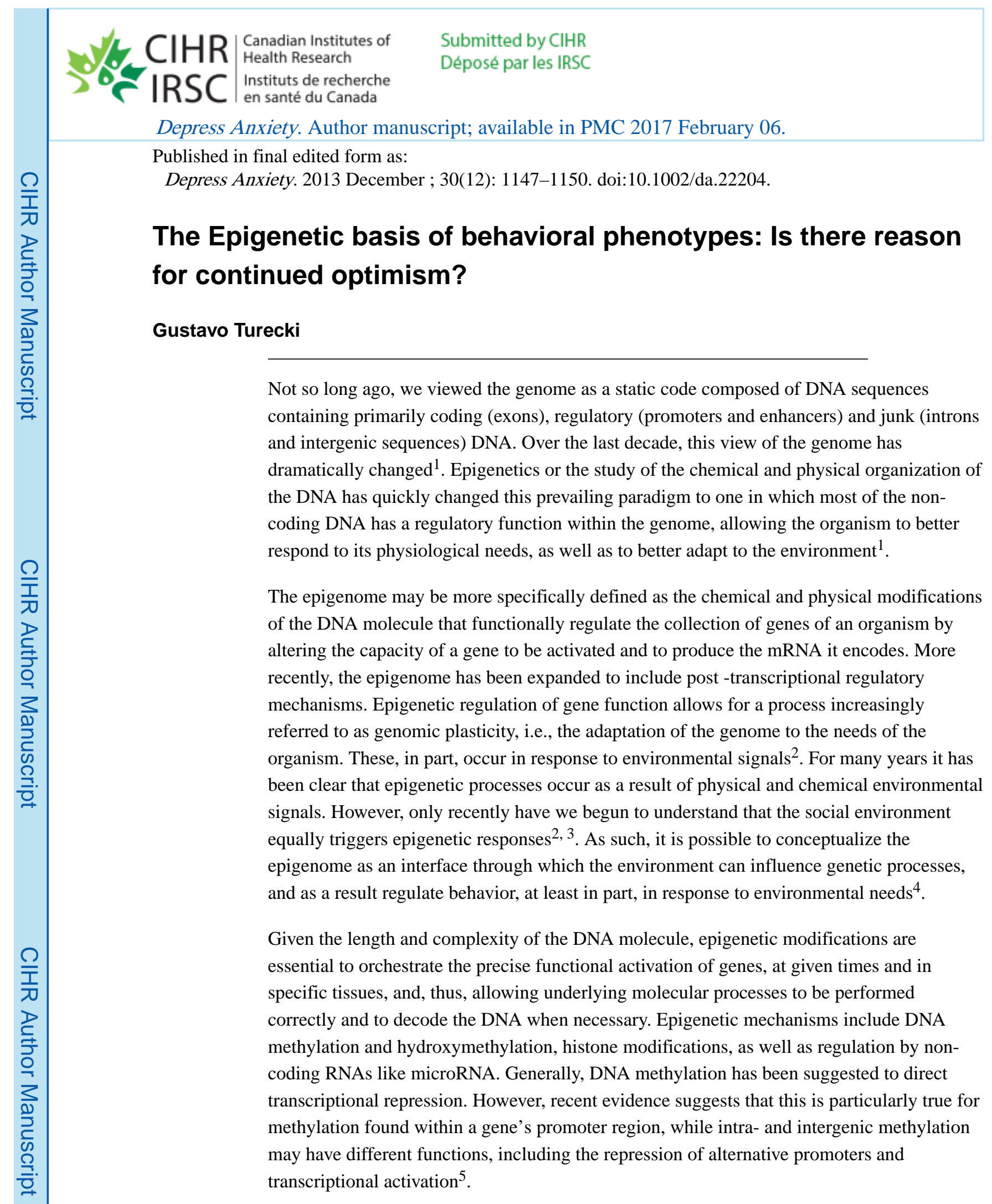

Not so long ago, we viewed the genome as a static code composed of DNA sequences containing primarily coding (exons), regulatory (promoters and enhancers) and junk (introns and intergenic sequences) DNA. Over the last decade, this view of the genome has dramatically changed ${ }^{1}$. Epigenetics or the study of the chemical and physical organization of the DNA has quickly changed this prevailing paradigm to one in which most of the noncoding DNA has a regulatory function within the genome, allowing the organism to better respond to its physiological needs, as well as to better adapt to the environment ${ }^{1}$.

The epigenome may be more specifically defined as the chemical and physical modifications of the DNA molecule that functionally regulate the collection of genes of an organism by altering the capacity of a gene to be activated and to produce the mRNA it encodes. More mechanisms. Epigenetic regulation of gene function allows for a process increasingly referred to as genomic plasticity, i.e., the adaptation of the genome to the needs of the organism. These, in part, occur in response to environmental signals ${ }^{2}$. For many years it has been clear that epigenetic processes occur as a result of physical and chemical environmental signals. However, only recently have we begun to understand that the social environment equally triggers epigenetic responses ${ }^{2,3}$. As such, it is possible to conceptualize the epigenome as an interface through which the environment can influence genetic processes, and as a result regulate behavior, at least in part, in response to environmental needs ${ }^{4}$.

Given the length and complexity of the DNA molecule, epigenetic modifications are essential to orchestrate the precise functional activation of genes, at given times and in specific tissues, and, thus, allowing underlying molecular processes to be performed correctly and to decode the DNA when necessary. Epigenetic mechanisms include DNA methylation and hydroxymethylation, histone modifications, as well as regulation by noncoding RNAs like microRNA. Generally, DNA methylation has been suggested to direct transcriptional repression. However, recent evidence suggests that this is particularly true for methylation found within a gene's promoter region, while intra- and intergenic methylation transcriptional activation ${ }^{5}$.

Correspondence: Gustavo Turecki MD PhD, McGill Group for Suicide Studies, Douglas Mental Health Institute, McGill University, 6875 LaSalle blvd, Montreal, QCH4H 1R3, Canada, gustavo.turecki@mcgill.ca. 


\section{Behavioral epigenetics}

Boosted by pioneering rodent work suggesting that the hypothalamus-pituitary-adrenal (HPA) axis is epigenetically programmed by the early-life environment ${ }^{6}$, and that these mechanisms are evolutionarily conserved ${ }^{7}$, epigenetics quickly became the holy grail of mental health researchers. After decades of infighting between psychosocial researchers and researchers of more biological inclination, as to whether mental illness results primarily from nature or nurture influences, epigenetics provided mental health researchers with a theoretical framework and a scientific paradigm capable of addressing previously conflicting and irreconcilable viewpoints on the causes of mental illnesses. Not surprisingly, there has been much enthusiasm for epigenetic studies of mental illness and related behavioral phenotypes, and we have seen an exponential growth in the number of studies investigating epigenetic factors in mental disorders. Almost a decade has passed since we began investigating the epigenetics of behavioral phenotypes, and we should ask ourselves whether there is reason for continued enthusiasm.

To address this question, let's consider the HPA stress response system, which is probably the most widely investigated biological system in terms of epigenetic regulation associated with behavioral phenotypes. The influence of the early-life environment on the regulation of the HPA is substantiated by groundbreaking findings from animal studies. A powerful model of early-life environmental influences on biological systems, and in particular on the activity of the HPA axis, is provided by the investigation of naturally-occurring variations in maternal care in rats. Variation in maternal care as measured by levels of licking and grooming (LG) of pups by their mothers, stably associates with brain expression levels of critical HPA genes and behavioral characteristics in offspring. For instance, rats raised by low LG mothers exhibit depressive-like behaviors ${ }^{8}$ associated with altered HPA axis feedback ${ }^{9}$ and low glucocorticoid receptor (GR) mRNA hippocampal levels ${ }^{9}$, and these characteristics are stable to adulthood. Moreover, cross-fostering studies report that these behavioral and molecular modifications are reversed when pups raised by low LG mothers are transferred to the care of high LG mothers within the first week of life ${ }^{8,9}$.

As the HPA and behavioral trait programming by maternal behavior can be reversed by cross-fostering and is temporally stable, it is clear that key biological systems are regulated by early-life environmental factors. At the molecular level, these effects are determined through DNA methylation changes. Specifically, in offspring raised by low LG rat mothers, CpG methylation levels in the GR exon 17 promoter (a variant of the GR gene that is important for its expression in the hippocampus) are significantly increased compared to offspring raised by high LG mothers. More importantly, in offspring raised by low LG mothers, the binding site for a $\mathrm{CpG}$ located in the $5^{\prime}$ end of nerve growth factor-inducible protein A (NGFI-A; a transcription factor that drives GR expression in the hippocampus) is almost $100 \%$ methylated whereas in offspring from high LG mothers the same site is almost not methylated ${ }^{6}$. Interestingly, follow-up studies in high and low LG rats showed that DNA methylation levels are also increased in the promoters of other non-coding first exons of the GR gene which were associated with transcriptional changes ${ }^{10}$ suggesting that the whole GR locus may be poised for epigenetic regulation by environmental social factors. 


\section{Translating animal findings to humans}

These pioneering animal studies encouraged further investigations into how these results may translate to humans. In collaboration with the scientists involved in the original rodent work, we conducted the first studies investigating the GR gene methylation patterns in association with variation in the quality of the early-life environment. Studying postmortem hippocampal tissue from individuals who died by suicide and had a history of severe childhood maltreatment (defined as having histories of severe childhood sexual, physical abuse, and/or parental neglect), we investigated the patterns of methylation in the promoter of the $1_{\mathrm{F}}$ promoter variant, which is homologous to the $1_{7}$ rodent variant. We observed results that were consistent with the rodent work, i.e., less favorable environments associate with decreased expression of the total GR gene and of the $1_{\mathrm{F}}$ variant, and increased methylation of the $1_{\mathrm{F}}$ promoter, which was observed particularly in areas where the transcription factor NGFI-A, binds. This transcription factor is important for driving the expression of the GR gene in the hippocampus, among other tissues ${ }^{6}$. Follow-up work suggested that, in addition to the $1_{\mathrm{F}}$ variant, the early-life environment regulates the expression of the $1_{\mathrm{B}}, 1_{\mathrm{C}}$ and $1_{\mathrm{H}}$ variants in the hippocampus, but not in the anterior cingulate cortex, a cortical brain region that is also important in the regulation of the HPA axis. The parallel findings between animal and human studies suggest that evolutionarily conserved mechanisms are involved in the regulation of stress systems such as the HPA to early environmental cues. One possible interpretation of these findings is that the organism adjusts levels of alertness according to early-life environmental signals. As such, if the environment is hostile, the organism may regulate its major stress response system to remain constantly vigilant, whereas if the environment is more favorable, the organism may afford to be less alert. Evidence that these mechanisms may indeed be conserved between species comes from comparative work between human and rodent in methylation patterns in a $6.5 \mathrm{M}$ base-pair sequence centered around the $\mathrm{GR}^{7}$.

\section{Replication?}

Since these initial findings, a number of groups have conducted studies investigating methylation patterns of the GR gene inrelation to early-life unfavorable experiences. Although these studies have primarily used peripheral tissues and have investigated diverse measures of negative environmental experiences and have not always studied the same GR sequences, nevertheless their results are encouraging. This is because there is general consistency between these studies in outcome direction, i.e., they found results suggesting increased methylation in individuals exposed to less favorable environment measures. Investigating GR $1_{\mathrm{F}}$ promoter methylation in cord blood mononuclear cells from threemonth children who were exposed to maternal mood changes during the first trimester of pregnancy, Berliner et al ${ }^{11}$ observed increased methylation at an NGFI-A binding site in children of depressed mothers. Interestingly, increased methylation was associated with increased salivary cortical stress responses in these children when they were exposed to a non-noxious stressor. Proud et al ${ }^{12}$ investigated the association between a history of childhood maltreatment and GR $1_{\mathrm{F}}$ promoter methylation in peripheral DNA samples from adult patients with borderline personality disorder and major depression. They found increased DNA methylation in individuals with histories of childhood abuse and a positive 
correlation between severity and number of maltreatment types with GR $1_{\mathrm{F}}$ methylation. These results are consistent with increased methylation found at the GR gene in borderline patients by Dammann et al ${ }^{13}$. More recently, Perroud et al ${ }^{14}$ also reported increased GR methylationin individuals with bipolar disorder. Using a population-based cohort, Tyrka et al ${ }^{15}$ investigated the methylation status of the GR $1_{F}$ promoter in DNA obtained from peripheral blood samples. They found increased $1_{\mathrm{F}}$ promoter methylation in individuals who had histories of childhood maltreatment and parental neglect. Interestingly, their results suggested a functional relationship between peripheral GR methylation and cortisol responses as they found a negative correlation between methylation levels at certain $\mathrm{CpG}$ sites and cortisol response to the dexamethasone suppression test. While it is unclear if these findings reflect direct or proxy relationships, they are consistent with results observed in human postmortem and animal studies ${ }^{16,17}$. The epigenetic state of the GR was also evaluated in a study of 10-19 year-olds whose mothers were assessed retrospectively for a history of intimate partner violence ${ }^{18}$. Results indicated that such violence, when experienced by the mother during, but not before or after pregnancy, associates in the children with increased methylation in the promoter region of the exon $1_{\mathrm{F}}$, as measured in whole blood cells, suggesting that prenatal stress may play a role in the regulation of GR methylation. These results are similar to those from two other recent studies ${ }^{19,}{ }^{20}$, both of which reported an association between maternal stress during pregnancy and GR methylation state in the child.

\section{Future directions}

Although performed in different tissues and clinical populations with diverse measures of unfavorable events, and not always assessing similar $\mathrm{CpG}$ sites and/or GR promoter sequences, these studies are remarkably consistent in the direction of their findings. Indeed, collectively these studies suggest that exposure to early-life stress and/or stressful environments associates with increased methylation in the GR gene. The effect of increased methylation in GR regulatory regions impacts GR gene expression levels ${ }^{16,17}$ and may thus have a functional impact on regulation of HPA activity, as it is the case in rodents ${ }^{23}$. The association of GR methylation patterns with peripheral markers of HPA axis activity is further evidence that this may be the case ${ }^{15,21,22}$ even though effects may be sexdependent ${ }^{21}$. While it is still early to draw definite conclusions, the findings discussed above are certainly encouraging and show a level of consistency not commonly observed in biological psychiatry. Therefore, there is sufficient reason for continued enthusiasmin the investigation of epigenetic factors in mental disorders, particularly as they mediate the effects of environmental influences on stress-response systems and behavior. However, there are many important questions that should be the focus of future studies. Among these, the field needs to better understand how exposure to adversity results in differential methylation, whether or not the nature of the stressor has an impact on methylation patterns and the developmental timing of methylation changes. In addition, studies should standardize methodologies to improve comparison of findings and facilitate multimember initiatives. 


\section{References}

1. Dunham I, et al. An integrated encyclopedia of DNA elements in the human genome. Nature. 2012; 489:57-74. [PubMed: 22955616]

2. Nagy C, Turecki G. Sensitive periods in epigenetics: bringing us closer to complex behavioral phenotypes. Epigenetic. 2012; 4:445-457.

3. Lamont B, Turecki G. The epigenetics of suicide: explaining the biological effects of early life environmental adversity. Arch Suicide Rees. 2010; 14:291-310.

4. Turecki G, Ernst C, Gallant F, Lamont B, Peshawar N. The neurodevelopment origins of suicidal behavior. Trends Neurosis. 2012; 35:14-23.

5. Maunakea AK, et al. Conserved role of intragenic DNA methylation in regulating alternative promoters. Nature. 2010; 466:253-257. [PubMed: 20613842]

6. Weaver IC, et al. Epigenetic programming by maternal behavior. Nat Neurosci. 2004; 7:847-854. [PubMed: 15220929]

7. Suderman M, et al. Conserved epigenetic sensitivity to early life experience in the rat and human hippocampus. Proc Natl Acad Sci U S A. 2012; 109(Suppl 2):17266-17272. [PubMed: 23045659]

8. Francis D, Diorio J, Liu D, Meaney MJ. Nongenomic transmission across generations of maternal behavior and stress responses in the rat. Science. 1999; 286:1155-1158. [PubMed: 10550053]

9. Liu D, et al. Maternal care, hippocampal glucocorticoid receptors, and hypothalamic-pituitaryadrenal responses to stress. Science. 1997; 277:1659-1662. [PubMed: 9287218]

10. McGowan PO, et al. Broad epigenetic signature of maternal care in the brain of adult rats. PLoS One. 2011; 6:e14739. [PubMed: 21386994]

11. Oberlander TF, et al. Prenatal exposure to maternal depression, neonatal methylation of human glucocorticoid receptor gene (NR3C1) and infant cortisol stress responses. Epigenetics. 2008; 3:97-106. [PubMed: 18536531]

12. Perroud N, et al. Increased methylation of glucocorticoid receptor gene (NR3C1) in adults with a history of childhood maltreatment: a link with the severity and type of trauma. Transl Psychiatry. 2011; 1:e59. [PubMed: 22832351]

13. Dammann G, et al. Increased DNA methylation of neuropsychiatric genes occurs in borderline personality disorder. Epigenetics. 2011; 6:1454-1462. [PubMed: 22139575]

14. Perroud N, et al. Childhood maltreatment and methylation of the glucocorticoid receptor gene NR3C1 in bipolar disorder. Br J Psychiatry. 2013

15. Tyrka AR, Price LH, Marsit C, Walters OC, Carpenter LL. Childhood adversity and epigenetic modulation of the leukocyte glucocorticoid receptor: preliminary findings in healthy adults. PLoS One. 2012; 7:e30148. [PubMed: 22295073]

16. Labonte B, et al. Differential glucocorticoid receptor exon 1(B), 1(C), and 1(H) expression and methylation in suicide completers with a history of childhood abuse. Biol Psychiatry. 2012; 72:4148. [PubMed: 22444201]

17. McGowan PO, et al. Epigenetic regulation of the glucocorticoid receptor in human brain associates with childhood abuse. Nat Neurosci. 2009; 12:342-348. [PubMed: 19234457]

18. Radtke KM, et al. Transgenerational impact of intimate partner violence on methylation in the promoter of the glucocorticoid receptor. Transl Psychiatry. 2011; 1:e21. [PubMed: 22832523]

19. Mulligan CJ, D’Errico NC, Stees J, Hughes DA. Methylation changes at NR3C1 in newborns associate with maternal prenatal stress exposure and newborn birth weight. Epigenetics. 2012; 7:853-857. [PubMed: 22810058]

20. Hompes T, et al. Investigating the influence of maternal cortisol and emotional state during pregnancy on the DNA methylation status of the glucocorticoid receptor gene (NR3C1) promoter region in cord blood. J Psychiatr Res. 2013; 47:880-891. [PubMed: 23566423]

21. Edelman S, et al. Epigenetic and genetic factors predict women's salivary cortisol following a threat to the social self. PLoS One. 2012; 7:e48597. [PubMed: 23155396]

22. de Rooij SR, et al. Associations between DNA methylation of a glucocorticoid receptor promoter and acute stress responses in a large healthy adult population are largely explained by lifestyle and educational differences. Psychoneuro endocrinology. 2012; 37:782-788. 
23. Meaney MJ, et al. Early environmental regulation of forebrain glucocorticoid receptor gene expression: implications for adrenocortical responses to stress. Developmental neuroscience. 1996; 18:49-72. [PubMed: 8840086] 